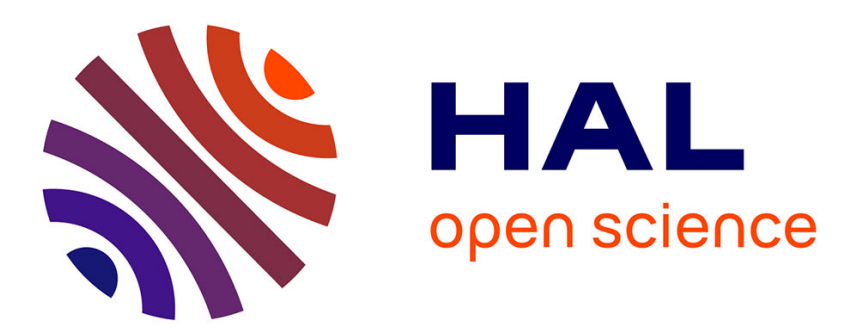

\title{
MESSENGER Observations of Planetary Ion Characteristics in the Vicinity of Kelvin-Helmholtz Vortices at Mercury
}

Sae Aizawa, Jim M. Raines, Dominique C. Delcourt, Naoki Terada, Nicolas André

\section{To cite this version:}

Sae Aizawa, Jim M. Raines, Dominique C. Delcourt, Naoki Terada, Nicolas André. MESSENGER Observations of Planetary Ion Characteristics in the Vicinity of Kelvin-Helmholtz Vortices at Mercury. Journal of Geophysical Research Space Physics, 2020, 125 (10), 10.1029/2020JA027871 . insu02968170

\section{HAL Id: insu-02968170 \\ https://hal-insu.archives-ouvertes.fr/insu-02968170}

Submitted on 15 Oct 2020

HAL is a multi-disciplinary open access archive for the deposit and dissemination of scientific research documents, whether they are published or not. The documents may come from teaching and research institutions in France or abroad, or from public or private research centers.
L'archive ouverte pluridisciplinaire HAL, est destinée au dépôt et à la diffusion de documents scientifiques de niveau recherche, publiés ou non, émanant des établissements d'enseignement et de recherche français ou étrangers, des laboratoires publics ou privés. 


\section{JGR Space Physics}

\section{RESEARCH ARTICLE 10.1029/2020JA027871 \\ Key Points: \\ - We investigate the statistical characteristics of planetary ions derived from MESSENGER FIPS \\ MESSENGER Observations of Planetary Ion Characteristics in the Vicinity of Kelvin-Helmholtz Vortices at Mercury} observations in the presence of $\mathrm{KH}$ waves

- Large counts in FIPS data and a decelerating signature are observed above $2.0 \mathrm{keV} / \mathrm{e}$ for $\mathrm{KH}$ events

- KH waves may play a role in the deceleration of planetary ions in the Hermean magnetosphere

Supporting Information:

- Supporting Information S1

- Table S1

Correspondence to:

S. Aizawa,

sae.aizawa@irap.omp.eu

Citation:

Aizawa, S., Raines, J. M., Delcourt, D., Terada, N., \& André, N. (2020). MESSENGER observations of planetary ion characteristics in the vicinity of Kelvin-Helmholtz vortices at Mercury. Journal of Geophysical Research: Space Physics, 125, e2020JA027871. https:// doi.org/10.1029/2020JA027871

Received 31 JAN 2020 Accepted 23 SEP 2020 Accepted article online 5 OCT 2020

\author{
Sae Aizawa ${ }^{1,2}$ (D), Jim M. Raines ${ }^{3}(\mathbb{D})$, Dominique Delcourt ${ }^{4}(\mathbb{D})$, Naoki Terada ${ }^{2}(\mathbb{D}$, \\ and Nicolas André ${ }^{1}$ iD \\ ${ }^{1}$ IRAP, CNRS-UPS-CNES, Toulouse, France, ${ }^{2}$ Department of Geophysics, Graduate School of Science, Tohoku University, \\ Sendai, Japan, ${ }^{3}$ Department of Climate and Space Sciences and Engineering, University of Michigan, Ann Arbor, MI, \\ USA, ${ }^{4}$ LPC2E, Université Orléans-CNRS, Orléans, France
}

\begin{abstract}
The MErcury Surface, Space ENvironment, GEochemistry, and Ranging (MESSENGER) spacecraft regularly observed the magnetospheric flanks of Mercury during its orbital phase. Data from the Magnetometer (MAG) and the Fast Imaging Plasma Spectrometer (FIPS) allow us to investigate the statistical properties of planetary ions $\left(\mathrm{Na}^{+}\right)$in the presence of Kelvin-Helmholtz $(\mathrm{KH})$ waves at the duskside magnetopause. We collect the data from orbits with clear signatures of $\mathrm{KH}$ waves under northward interplanetary magnetic field, as well as from adjacent orbits that do not have KH signatures, and we compare the energy characteristics between the $\mathrm{KH}$ and non- $\mathrm{KH}$ events. Although low planetary counts in FIPS data make the comparison of these characteristics difficult, we find that in the presence of $\mathrm{KH}$ waves: (1) large counts of planetary ions are observed and (2) differences in $\mathrm{Na}^{+}$energy spectra are only seen inside the magnetosphere, where they show a deceleration signature for ions with energies above $2.0 \mathrm{keV} / \mathrm{e}$. These results suggest that planetary ions are not escaping from the magnetosphere and that electric field structures related to $\mathrm{KH}$ waves can decelerate planetary ions originating from the magnetotail region. The understanding of the energy distribution of planetary ions in the magnetospheric flanks of Mercury is important for a better understanding of plasma convection in the magnetosphere.
\end{abstract}

\section{Introduction}

The well-known magnetohydrodynamic (MHD) Kelvin-Helmholtz (KH) instability is considered as an important phenomenon for the solar wind penetrating planetary magnetospheres when the interplanetary magnetic field (IMF) is parallel to the planetary magnetic field. Generally, it occurs when there is a velocity shear between two adjacent plasma flows and forms a rolled-up $\mathrm{KH}$ vortex when fully developed. Around magnetized planets, the magnetopause is a boundary layer (BL) between the solar wind and magnetospheric plasmas with a strong velocity shear and the $\mathrm{KH}$ instability is expected to develop even if plasma is collisionless (Hasegawa et al., 2004). The KH vortices around magnetized planets play roles in the transport of mass and momentum of plasma, resulting in mixing two different plasmas (Fujimoto \& Terasawa, 1994; Miura, 1984). It can also be a trigger of the magnetic reconnection inside the vortex that leads to plasma mixing across the shear layer (e.g., Nakamura \& Fujimoto, 2006; Nykyri \& Otto, 2001).

At Earth's magnetopause, there are many observational reports of $\mathrm{KH}$ waves provided by various spacecraft (e.g., Chen \& Kivelson, 1993; Fujimoto et al., 2003; Hasegawa et al., 2004; Hwang et al., 2011; Mozer et al., 1994; Yan et al., 2014). KH waves at Earth have been observed on both dawn and dusk sides. As for Mercury, several studies reported evidences of KH vortices in MErcury Surface, Space ENvironment, GEochemistry, and Ranging (MESSENGER) data (Gershman et al., 2015; Liljeblad et al., 2014, 2016; Slavin et al., 2008; Sundberg et al., 2011, 2012). Unlike in cases at Earth, KH waves at Mercury have been observed mainly in duskside magnetospheric flanks, and its occurrence is $95 \%$ of all observations at Mercury. Many authors have suggested that this strong asymmetry on the KH occurrence at Mercury may be due to the kinetic effect or the lack of large-scale laminar flow on the dawnside (Liljeblad et al., 2014; Paral \& Rankin, 2013). In particular, because of the small size of Mercury's magnetosphere compared to that of Earth, the spatial and temporal scale of physical phenomena around Mercury and those of the ion 
gyration motion can be comparable; thus, the gyration motion of particles and the variation of fields may affect each other. Gershman et al. (2015) reported that the observed frequency of KH waves on the dusk side is modified by the $\mathrm{Na}^{+}$gyrofrequency. Gingell et al. (2015) examined the effect of the gyration motion of planetary sodium ions using hybrid simulations, and they revealed that the gyroresonance between the $\mathrm{KH}$ instability and the ion motion leads to a strong asymmetry for the growth of the $\mathrm{KH}$ instability between the dawn and dusk sides. In addition, Aizawa et al. (2018) investigated the particle energization in the $\mathrm{KH}$ vortex using test-particle tracing technique and MHD simulation and revealed that the planetary ions can be nonadiabatically accelerated/decelerated depending on the energy that the particle experiences during its gyration motion. They suggested that the electric field variations due to the development of the $\mathrm{KH}$ instability can be responsible for particle acceleration in the magnetospheric flanks of Mercury.

The present study focuses on the energy distribution of planetary ions in the magnetospheric flanks where the macroscopic instability and microscopic ion kinetics affect each other. We investigate the effect of the presence of $\mathrm{KH}$ waves on the acceleration/deceleration of planetary ions. We use the data set from the Fast Imaging Plasma Spectrometer (FIPS) and the Magnetometer (MAG) instruments onboard the MESSENGER spacecraft. In section 2, we first describe the criteria for the data selection and the method of analysis. The results obtained from a case study and a statistical survey are presented in section 3 and discussed in section 4. Section 5 summarizes our conclusions.

\section{Data Selection and Analysis}

The MESSENGER spacecraft, the first Mercury orbiting spacecraft, was launched in 2004 and stayed in orbit from 18 March 2011 until 30 April 2015. Although MESSENGER payload was mostly dedicated to planetology studies, the plasma environment around Mercury has been investigated using MAG to measure the ambient magnetic field with a time resolution of $20 \mathrm{~s}^{-1}$ and FIPS to measure the composition of heavy ions with an energy-per-charge range from $50 \mathrm{eV} / \mathrm{e}$ up to $13.3 \mathrm{keV} / \mathrm{e}$. FIPS is a time-of-flight plasma mass spectrometer, and it can distinguish $\mathrm{H}^{+}, \mathrm{He}^{+}, \mathrm{He}^{2+}, \mathrm{O}^{+}$group (mass-per-charge 16-20) and $\mathrm{Na}^{+}$group (mass-percharge 21-30). FIPS measured the planetary ions that are expelled from the exosphere after photoionization. FIPS provided information on Mercury's magnetosphere and surrounding space plasma environment with a time resolution of $\sim 10 \mathrm{~s}$. Since MESSENGER was protected from the harsh conditions close to the Sun using sunshield, the FIPS instrument had a limited field of view (FOV) of only $1.4 \pi$ sr with an angular resolution of $\sim 15^{\circ}$ (e.g., Gershman et al., 2013; Raines et al., 2013). In this study, the data from both FIPS and MAG in the entire orbital phase of MESSENGER have been analyzed.

Like in previous studies, $\mathrm{KH}$ waves were identified using the magnetic field data. Here, we selected all data featuring fluctuations near the duskside magnetopause crossing point. The detailed data selection procedure consists of the following steps:

1. In order to restrict the certain frequency of $\mathrm{KH}$ waves as previously reported, a band-pass filter is applied for the selection of the oscillation between 0.01 and $0.1 \mathrm{~Hz}$ (e.g., Gershman et al., 2015).

2. Other oscillations such as flux transfer events (FTE), mirror waves, or magnetopause motions (e.g., Liljeblad et al., 2014) are removed. FTE and other oscillations are checked by visual inspection of the reversals of the $y$ component of the magnetic field (e.g., Slavin et al., 2010).

3. The periodicity of $\mathrm{KH}$ waves is checked by visual inspection.

Once $\mathrm{KH}$ events were identified, we record $\mathrm{Na}^{+}$group counts in FIPS data and examine their energy distribution. To build our statistics and compare $\mathrm{KH}$ and non- $\mathrm{KH}$ cases, we consider for each $\mathrm{KH}$ event two preceding and two subsequent orbits of MESSENGER as non-KH events. (Note that some of them may exhibit $\mathrm{KH}$ waves. In that case, those events are considered as $\mathrm{KH}$ events. Please see the supporting information). Note here that MESSENGER had a 12-hr orbit in the period from 18 March 2011 to 25 April 2012 and an 8-hr orbit in the rest of the orbital phase of the mission and that the azimuthal drift from one orbit to the other is small. We assume that the same region had been observed between $\mathrm{KH}$ and non- $\mathrm{KH}$ cases.

The energy distribution of planetary ions is derived by summing $\mathrm{Na}^{+}$group ion phase space density (PSD) in time. This indicates that the energy distribution is highly dependent on its time range and total counts in FIPS. In this study, two different normalizations are applied to derive the plasma properties. One is done by using the total PSD value over all energy channels and the other by using the PSD value at the 
particular energy of $1.0 \mathrm{keV} / \mathrm{e}$ (or nearest energy when FIPS does not have counts at $1.0 \mathrm{keV} / \mathrm{e}$ ). These two normalizations proved necessary to remove the artifacts in the properties of the $\mathrm{Na}^{+}$PSD, namely, differences due just to the ambient density (or flux) of the particles and not differences due to the energization that are the focus of the present study. Note that cases having only one count within a given energy bin for the whole summation period were removed because the data uncertainty becomes automatically $100 \%$. The error bar in each event is calculated by $\varepsilon=f \frac{\sqrt{N}}{N}$, where $f$ is the PSD of planetary ions and $N$ the FIPS counts. Since the results obtained from our two ways of normalization do not show significant differences, the results of the normalization based on the total PSD value will be shown hereafter for simplicity and readability.

In addition, the FIPS clock angle (i.e., the angle between the $Y$ axis in the Mercury-Solar-Orbital [MSO] coordinate system $[X$ is directed from the center of the planet toward the Sun, $Z$ is normal to Mercury's orbital plane, and $Y$ is taken to complete the right hand system] and the FIPS boresight vector) also is an important factor for the $\mathrm{Na}^{+}$PSD behavior. If FIPS has a completely different clock angle between the considered orbit and the adjacent one, detected particles should come from a different region due to the limited FOV, and thus, it makes difficult to compare the plasma properties. Accordingly, the data reported in this study were all recorded within the same clock angle, namely, between $180^{\circ}$ and $270^{\circ}$ which is the most significant statistical range for our study.

Since some previous studies have already provided a list of identified $\mathrm{KH}$ events obtained from MESSENGER observations (Gershman et al., 2015; Sundberg et al., 2012), we first consider the nightsideduskside events (i.e., observed between 18:00 and 21:00 local time [LT]) extracted from their list (hereinafter referred to as Sundberg-Gershman [SG] events). Then we separately analyzed all data obtained during crossings of the nightside-duskside magnetopause observed between 19:00 and 21:00 LT and $\pm 30^{\circ}$ of the latitude from the equator during the whole MESSENGER orbital phase. Note that the southward IMF cases are excluded in this study because it is more difficult to identify $\mathrm{KH}$ waves and of its low occurrence at Mercury under that configuration (Liljeblad et al., 2014).

\section{Results}

\subsection{Case Study}

Sundberg et al. (2012) and Gershman et al. (2015) analyzed MESSENGER MAG data under northward IMF and identified KH events. They identified $25 \mathrm{KH}$ events in the dayside (between 14:00 and 16:00 LT, not analyzed in this study) and 34 in the nightside (between 18:00 and 21:00 LT). One of them observed on Orbit Number 1179 exhibits a typical KH signature with enough FIPS counts as is illustrated in Figure 1. Here the MSO coordinate system is used. As shown in the trajectory on the left, MESSENGER was moving from the southern hemisphere to the northern hemisphere. On the right, the top panel is the proton energy spectrogram in units of flux $\left(\mathrm{s}^{-1} \mathrm{~cm}^{-2} \mathrm{sr}^{-1} \mathrm{kV}^{-1}\right)$, each vertical time step corresponds to one full FIPS scan over a period of $10 \mathrm{~s}$. The second panel is the $\mathrm{Na}^{+}$group ions' energy spectrogram in the PSD units $\left(\mathrm{s}^{3} \mathrm{~km}^{-6}\right)$. The third panel shows the total counts for $\mathrm{Na}^{+}$group ion for each FIPS scan. The remaining four panels are from MAG, showing $B x, B y, B z$, and the magnetic field intensity, respectively. Here $\mathrm{KH}$ waves can be seen between 14:40 and 14:51 as delineated by two vertical gray lines. In order to compare the properties of the $\mathrm{Na}^{+} \mathrm{PSD}^{\mathrm{C}}$, we divide the region into three regions, that is, $10 \mathrm{~min}$ of magnetosheath (MSH) between 14:29 and 14:39, BL where we identify KH waves between $14: 40$ and 14:51, and 10 min of magnetosphere (MSP) between 14:52 and 15:02, following the identification of the magnetopause crossing at 14:51 (note that his boundary is defined here using the minimum variance analysis [e.g., Sonnerup \& Cahill, 1967] and visual inspection). Due to its limited FOV, FIPS does not observe many counts of $\mathrm{Na}^{+}$group ions in the MSH region, while some $\mathrm{Na}^{+}$group ions are detected in the BL and MSP regions.

Orbit Number 1180 is taken as the corresponding non-KH event for a comparison (see Figure 2). Three regions, $\mathrm{MSH}, \mathrm{BL}$, and $\mathrm{MSP}$, are defined based on the magnetopause crossing point. BL region has the same duration as in the $\mathrm{KH}$ event based on the magnetopause crossing point, and MSH and MSP regions are taken with 10 min of duration in the same manner for the KH case. In the case of Orbit 1180, the MSH, BL, and MSP are between 22:24 and 22:34, 22:35 and 22:46, and 22:46 and 22:56, respectively. 

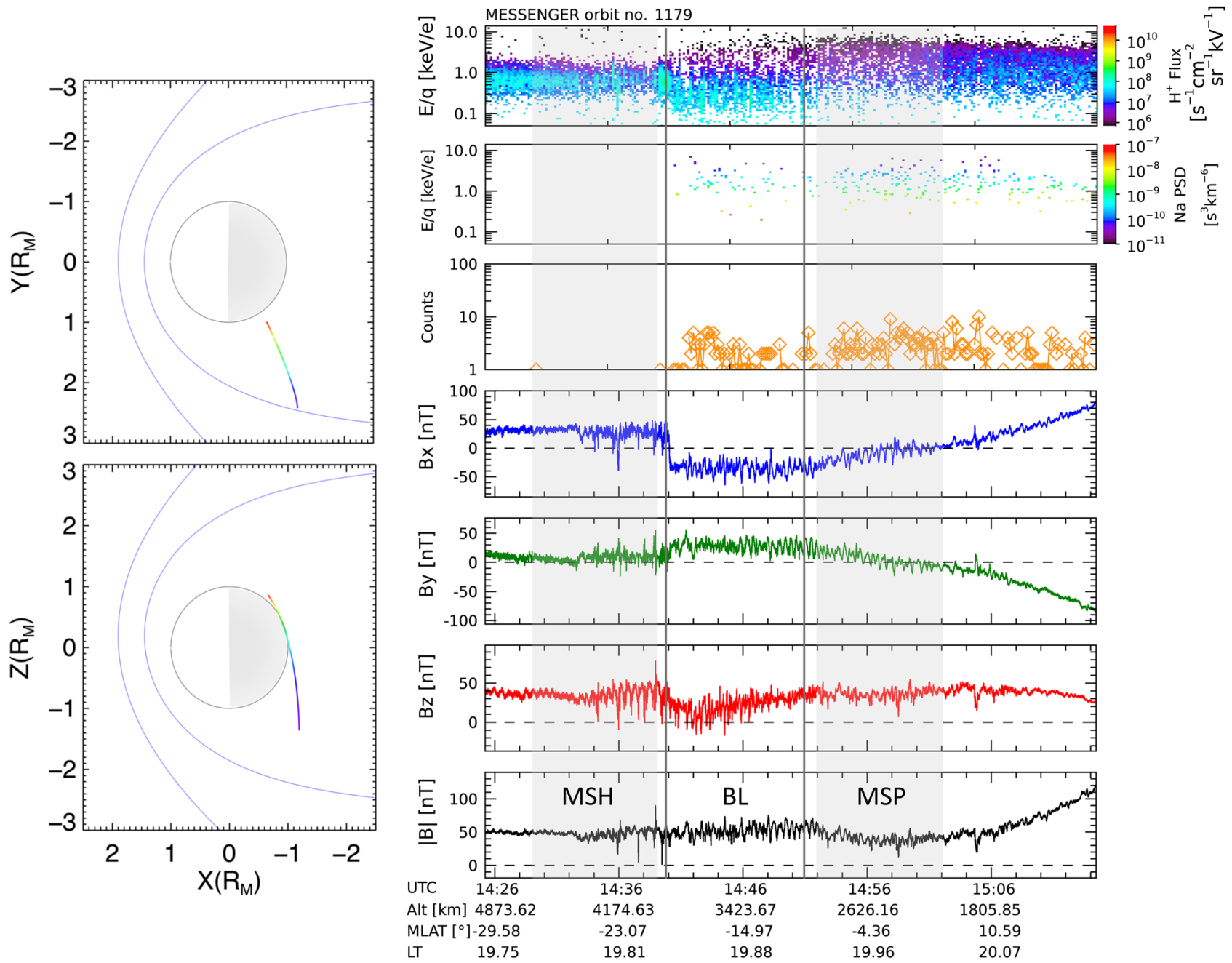

Figure 1. A typical KH signature taken from the SG events, observed during Orbit Number 1179. (right) From top to bottom: proton energy-time spectrogram in the unit of flux, $\mathrm{Na}^{+}$group energy-time spectrogram in the unit of PSD, total $\mathrm{Na}^{+}$group counts, time series of magnetic field component $(x, y$, and $z$; in MSO coordinates), and intensity. In the boundary region (BL), KH waves are seen between 14:40 and 14:51 as delineated by vertical gray lines. (left) The trajectory of MESSENGER in the $X$ - $Y$ plane (top) and in the $X$ - $Z$ plane (bottom), in MSO coordinates. Two blue lines represent the model of bowshock and

magnetopause by Slavin et al. (2009) and Shue et al. (1997), respectively. The parameters of the bowshock are given by $X_{0}=0.5 R_{M}, p=2.75 R_{M}$, and $\varepsilon=1.04$, and those of the magnetopause are given by $R_{S S}=1.45 R_{M}$ and $\alpha=0.5$ (Winslow et al., 2013). The spacecraft crossed the magnetopause in the southern hemisphere and approached the northern cusp region during the time interval displayed.

The obtained normalized $\mathrm{Na}^{+}$PSDs and count distribution in each region corresponding to the event of Figures 1 and 2 are presented in Figure 3. Colored symbols represent the KH case, and black symbols show the non-KH case. It is clearly seen that top panels of Figure 3 do not show clear differences between the KH and non- $\mathrm{KH}$ cases, and the $\mathrm{KH}$ case has larger counts in the energy around a few $\mathrm{keV}$. A significant difference here between $\mathrm{KH}$ and non-KH events is the total counts of the $\mathrm{Na}^{+}$group ions in FIPS data. For those orbits parameters like the spacecraft location, that is, LT, true anomaly angle (TAA for Mercury), latitude, and the FIPS clock angle are quite similar. In addition, the averaged $\mathrm{Bz}$ in MSH region, an important parameter for the growth of the $\mathrm{KH}$ instability, is $23.1 \mathrm{nT}$ for the $\mathrm{KH}$ and $13.3 \mathrm{nT}$ for the non-KH case; thus, the solar wind conditions can be assumed quite similar. However, there is such a large difference in the $\mathrm{Na}^{+}$ group counts despite these two orbits having mostly the same observation conditions. The difference between $\mathrm{Na}^{+}$energy-count distribution between both $\mathrm{KH}$ and non-KH events (hereinafter referred to as the $\mathrm{Na}^{+}$group count ratio) is shown in Figure 4. This ratio is obtained from the difference between counts in the $\mathrm{KH}$ and non-KH and then divided by the total non-KH counts in each region. Clearly, FIPS detected higher counts when $\mathrm{KH}$ waves are present. Also there are significant $\mathrm{Na}^{+}$group counts at the 

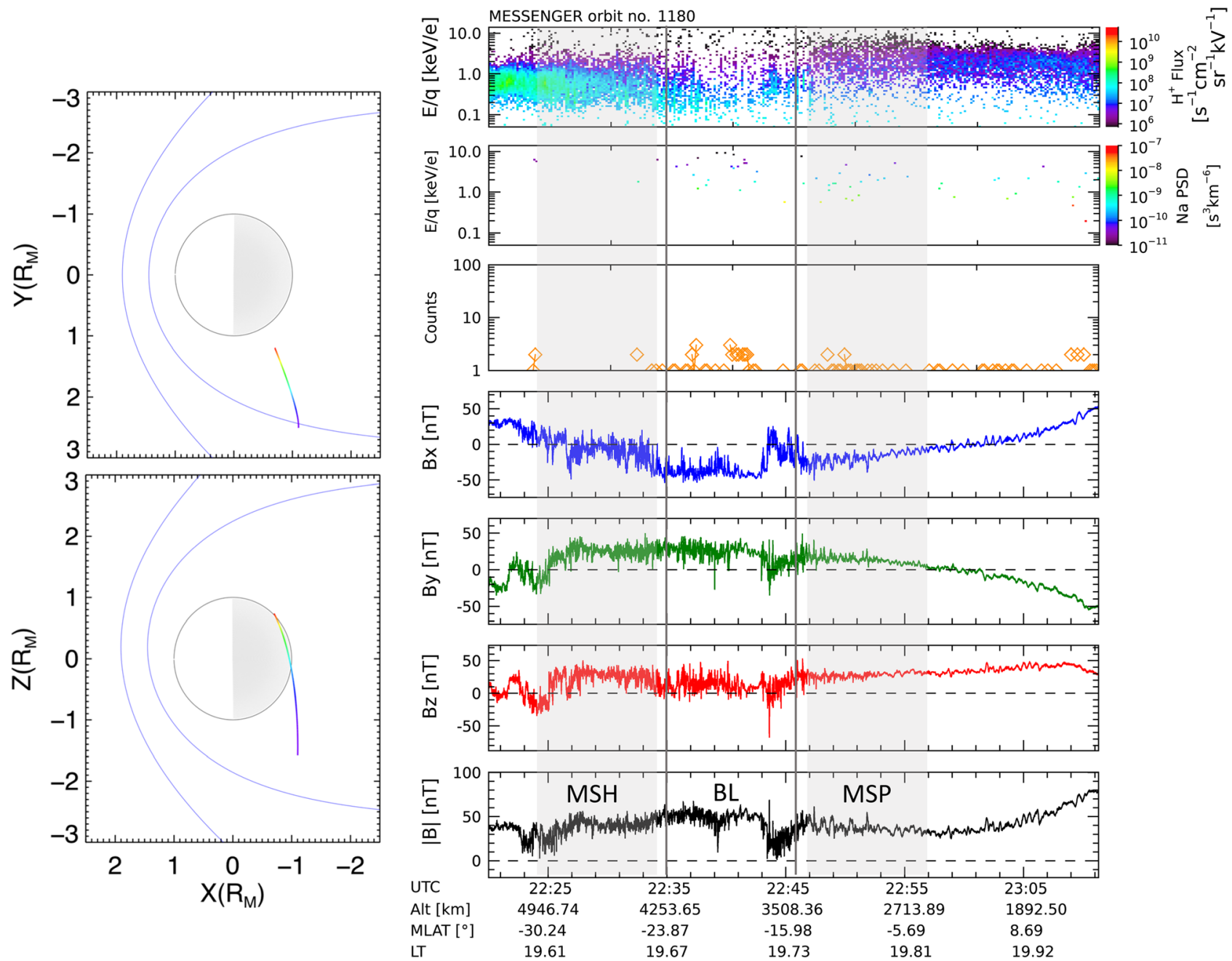

Figure 2. An adjacent non-KH case from Orbit Number 1180. The format of the figure is the same as the one of Figure 1. The spacecraft has a similar trajectory. In the case of Orbit Number 1180, the MSH, BL, and MSP are observed between 22:24 and 22:34, 22:35 and 22:46, and 22:46 and 22:56, respectively, based on the magnetopause crossing identified at 22:46 and the duration of the corresponding region for Orbit Number 1179 (see Figure 1).

lower energy, $\sim 1.0 \mathrm{keV} / \mathrm{e}$, while the counts are very low during non-KH events at this lower energy range. We will investigate later if these features are due to the acceleration or transport of ions by the $\mathrm{KH}$ instability. Indeed, picked up planetary ions of exospheric origin with a few $\mathrm{eV}$ could be observed around a few $\mathrm{keV}$ if they had undergone nonadiabatic acceleration due to the KH development (Aizawa et al., 2018).

\subsection{Statistical Survey}

Because KH waves are frequently detected and FIPS detected large counts of $\mathrm{Na}^{+}$group ions in the nightside-duskside of the magnetopause, all data recorded during magnetopause crossings for the entire orbital phase of MESSENGER have been analyzed, in addition to the $33 \mathrm{KH}$ events from the SG events. These 33 KH events were identified using the MAG data from April 2011 and February 2013 and are in the range of local time between 18:00 and 21:00. In this study, we first select all orbits with the magnetopause crossings within $\pm 30^{\circ}$ of latitude in the nightside (19:00-21:00 LT) events. We selected a smaller range in the local time so that we can discuss the influence only in the nightside magnetosphere. Although the SG events and events from the magnetopause crossings were analyzed separately, the results do not differ. In this section, we combine both cases and analyze them together.

From the SG event, 6 out of the remaining $33 \mathrm{KH}$ events are also removed due to the restricted range of the clock angle applied in the study. The remaining $27 \mathrm{KH}$ cases from the SG events are used in the statistical 

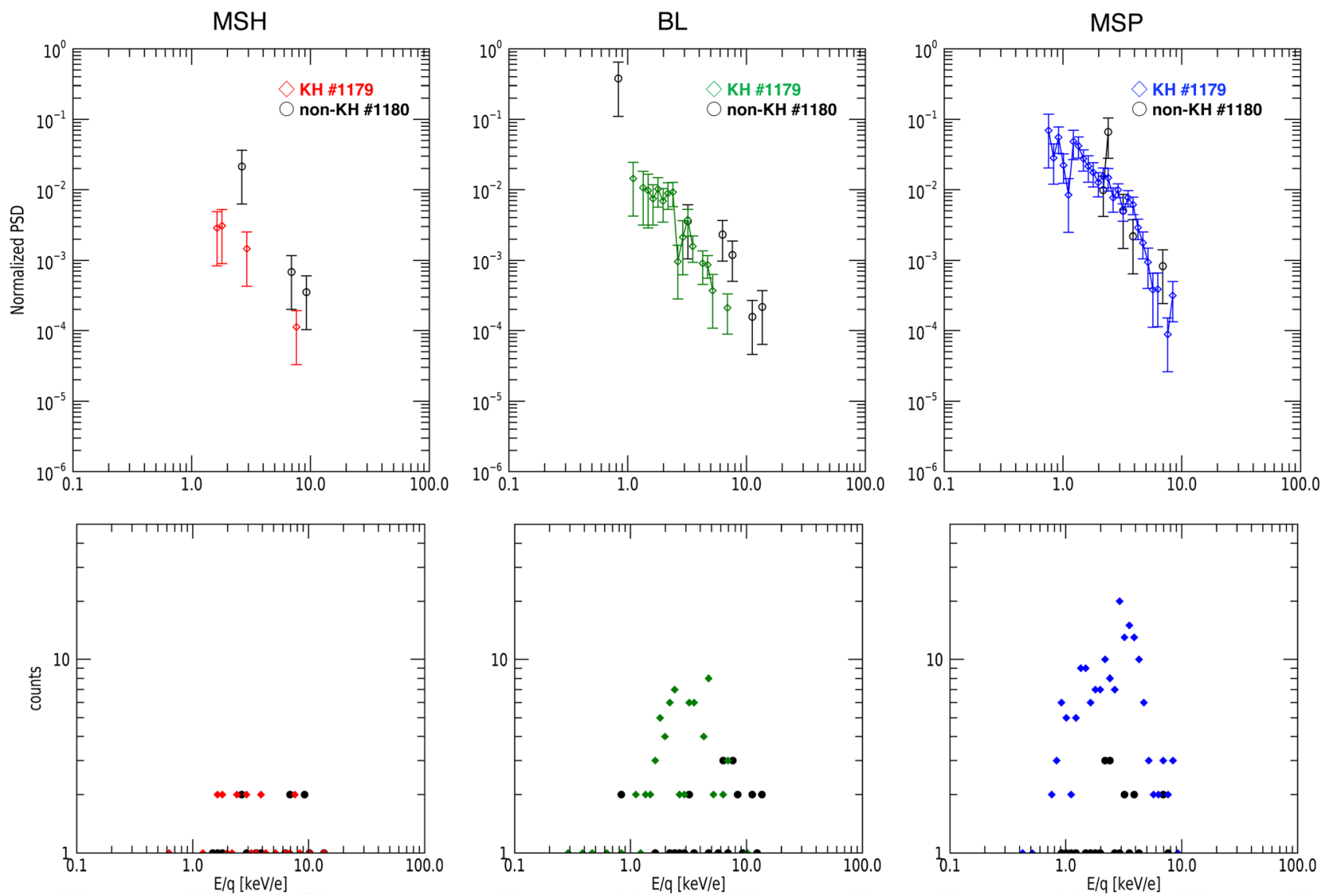

Figure 3. The properties of the $\mathrm{Na}^{+} \mathrm{PSD}$ (top row) and count distribution (bottom row) observed in each region (MSH, BL, and MSP; see text for details). Colored diamonds are used for the KH case observed during Orbit Number 1179, and black circles for the non-KH case observed during Orbit Number 1180. Error bars are calculated by $\varepsilon=f \frac{\sqrt{N}}{N}$, where $f$ is the phase space density of planetary ions and $N$ the FIPS counts.

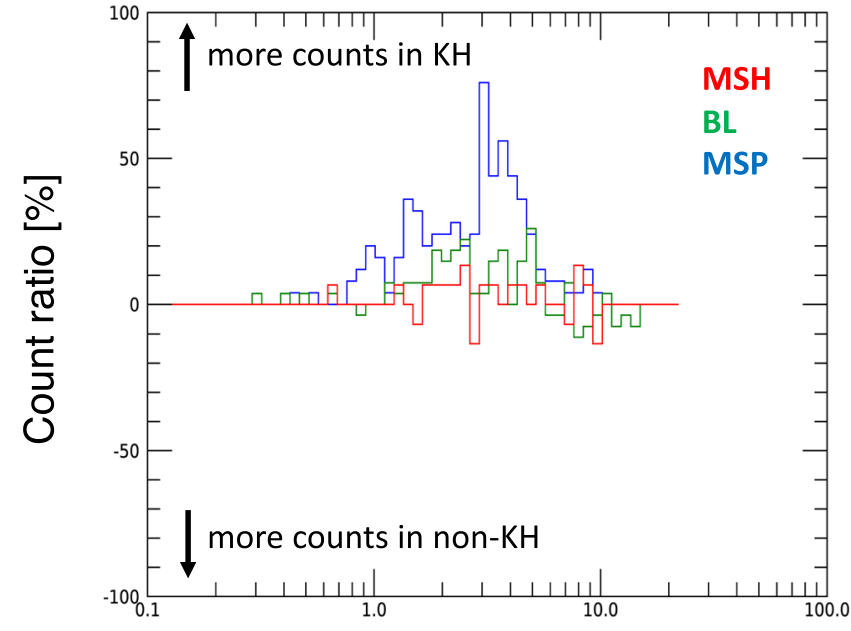

$\mathrm{E} / \mathrm{q}[\mathrm{keV} / \mathrm{e}]$

Figure 4. Histogram of the $\mathrm{Na}^{+}$group count ratio between $\mathrm{KH}$ (Orbit Number 1179) and non-KH (Orbit Number 1180) events. Positive values indicate that more counts are observed during $\mathrm{KH}$ events compared to non- $\mathrm{KH}$ ones. study. From the magnetopause crossing events, applying the criteria mentioned in section 2, we end up with $35 \mathrm{KH}$ events. The breakdown of our $35 \mathrm{KH}$ events is 8 of them in 2012, 12 in 2013, 14 in 2014, and 1 of them in 2015, respectively. After removing all invalid data (such as cases where $\mathrm{Na}^{+}$group counts are negligible, when the magnetic field points southward in the magnetosheath region, there are cases overlapped with the SG events, and the FIPS clock angle is between $180^{\circ}$ and $270^{\circ}$ ), we obtain $22 \mathrm{KH}$ events. It is worth noting that our selected KH events after 30 March 2013 have never been analyzed before. Combining the $27 \mathrm{KH}$ cases from the SG event and our $22 \mathrm{KH}$ cases, we finally analyze $49 \mathrm{KH}$ events in this statistical study (see tables in the supporting information). As for non-KH cases, we checked all two preceding and two subsequent orbits of MESSENGER as candidates for the corresponding $\mathrm{KH}$ event. We check all parameters such as averaged $\mathrm{Bz}$ in $\mathrm{MSH}$, counts in FIPS, and FIPS clock angle and confirm whether it is the $\mathrm{KH}$ event or not. In principle, each $\mathrm{KH}$ case has four corresponding non-KH cases. However, sometimes some of them show also $\mathrm{KH}$ waves. As non-KH cases, we have 72 cases in total. Details can be found in the supporting information.

Figure 5 shows the count distribution of $\mathrm{Na}^{+}$group ions in both the magnetosheath and the magnetosphere as identified in section 3 as a function 

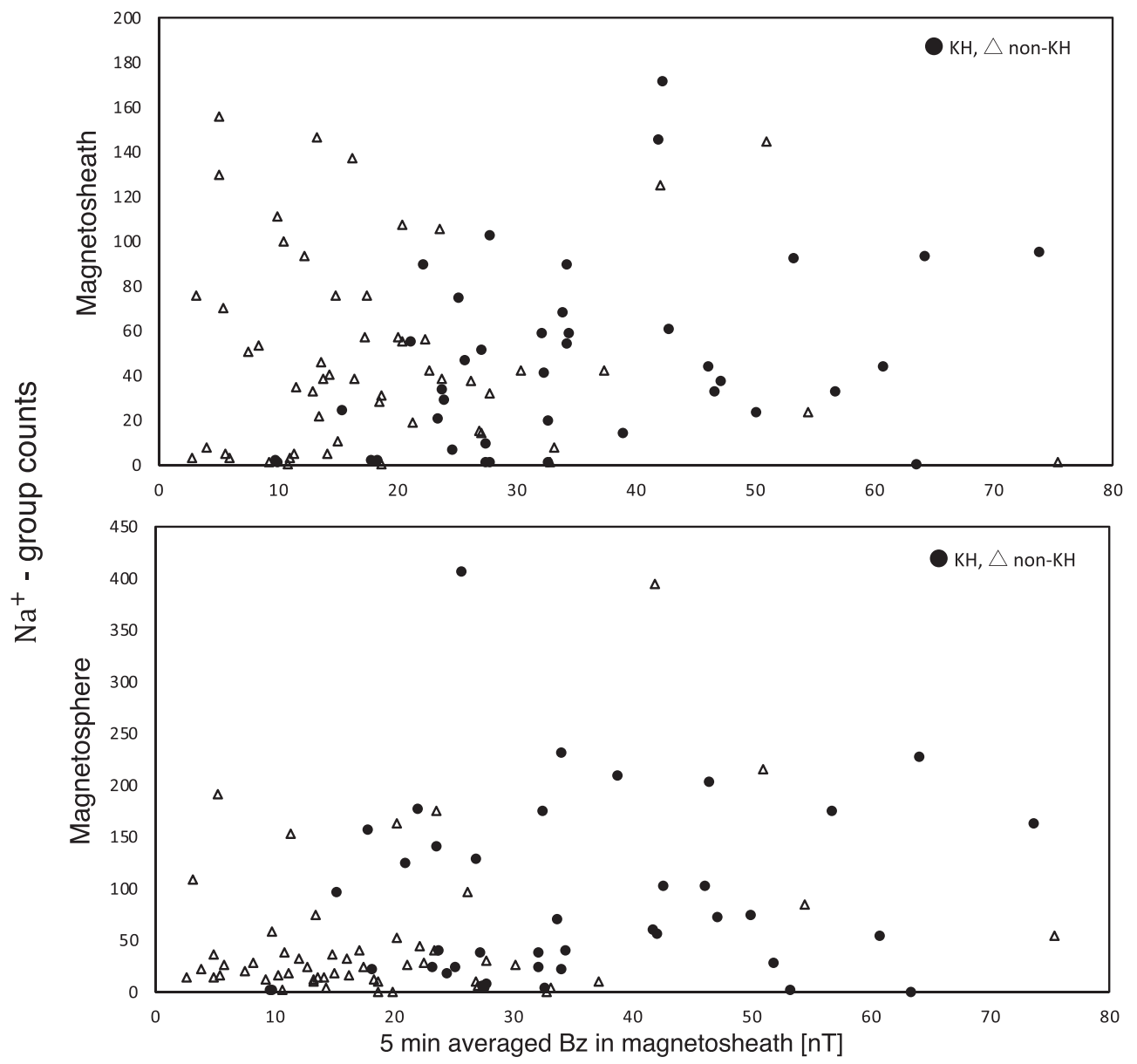

Figure 5. $\mathrm{Na}^{+}$count distribution observed in the magnetosheath (top) and magnetosphere (bottom) as a function of 5-min-averaged $\mathrm{Bz}$, in the magnetosheath. Black dots are used for $\mathrm{KH}$ events, and white triangles for non-KH events.

of the averaged $Z$ component of the magnetosheath magnetic field. We note that $\mathrm{KH}$ events have a magnetic field magnitude higher than $10 \mathrm{nT}$. In the linear theory of the growth of the KH instability, it is well known that the component of the magnetic field parallel to the wavenumber of the $\mathrm{KH}$ waves (here $B x$ and $B y$ ) inhibits the development of the instability (e.g., Elphic \& Ershkovich, 1984). Thus, the occurrence of the $\mathrm{KH}$ cases, that is, more cases at stronger $\mathrm{Bz}$, is understandable. On the other hand, $\mathrm{Na}^{+}$group counts in the magnetosheath do not show significant differences between the $\mathrm{KH}$ and non-KH cases while those in the magnetosphere show the large counts when the $\mathrm{KH}$ waves are present. The $\mathrm{Na}^{+}$group counts in $\mathrm{KH}$ cases do not show any significant dependence on the $B z$.

Then, in order to discuss the property of $\mathrm{Na}^{+}$group ions between the $\mathrm{KH}$ and non-KH cases, we check the pair of events. Out of $49 \mathrm{KH}$ cases, we finally obtained 16 cases with adjacent non-KH events. The quantitative analysis of the properties of the $\mathrm{Na}^{+}$PSD for these 16 cases is presented in Figure 6. First, we obtained the properties for each paired cases and then took the average of 16 cases. It is stressed here that the shape of the properties should be compared because the PSD value is normalized. In Figure 6, the properties in both the MSH and BL regions do not show significant differences between the $\mathrm{KH}$ and non-KH cases while a large difference for the $\mathrm{Na}^{+}$PSD between $\mathrm{KH}$ and non-KH events in the MSP region. Although the property in the energy below $0.7 \mathrm{keV}$ is quite similar between the $\mathrm{KH}$ and non- $\mathrm{KH}$ cases, we see steeper structure when $\mathrm{KH}$ waves are present (see blue line in Figure 6). It indicates that the properties of $\mathrm{Na}^{+}$group ions, namely, the distribution of planetary ions, are significantly different among them. Most notably, it can be seen in this figure that, above $2.0 \mathrm{keV} / \mathrm{e}$, planetary ions are decelerated when $\mathrm{KH}$ waves are recorded. It should be also mentioned here that there is negligible acceleration around $1 \mathrm{keV}$. In addition to this quantitative $\mathrm{Na}^{+}$ 
$\mathrm{MSH}$

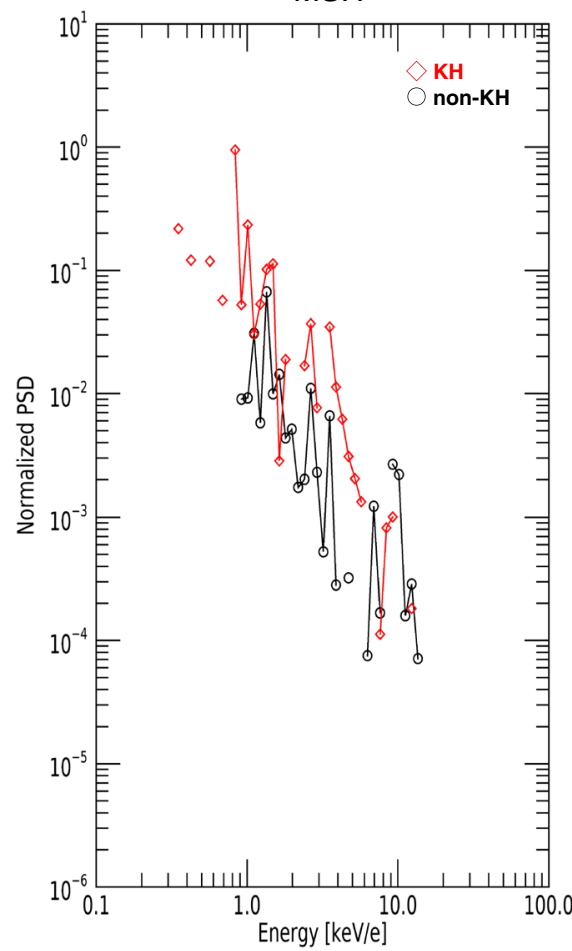

$\mathrm{BL}$

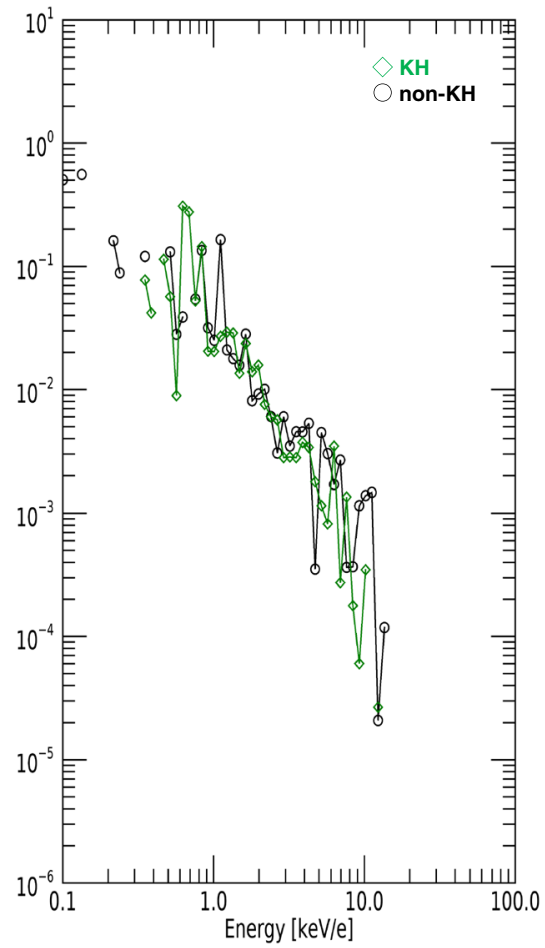

MSP

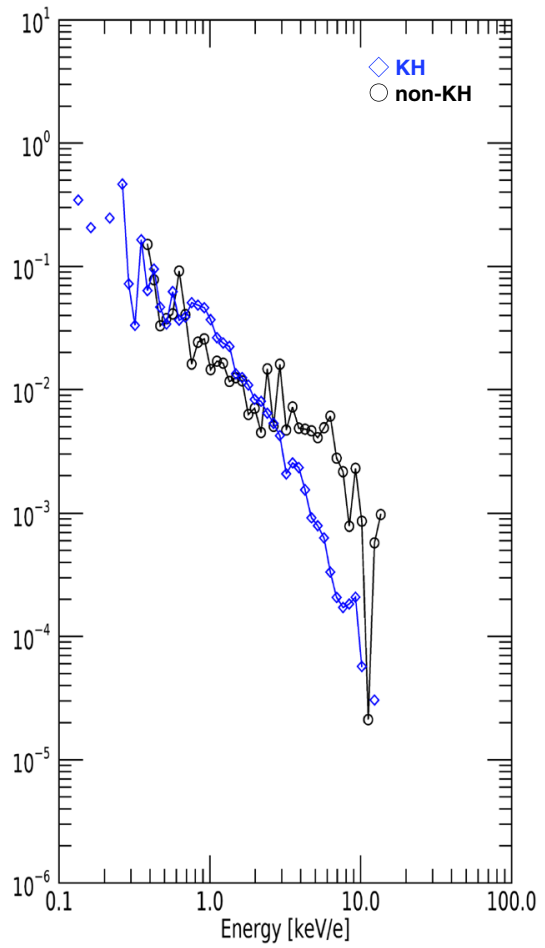

Figure 6. Averaged $\mathrm{Na}^{+}$PSD behaviors and count distribution observed in each region (MSH, BL, and MSP; see text for details) for the 16 paired KH events identified in the text. Colored diamonds are used for $\mathrm{KH}$ cases, and black circles for non-KH cases.

PSD behavior, the result of the $\mathrm{Na}^{+}$group counts in FIPS is shown in Figure 7. Although the count ratio can be low due to the fact that we take the average, the histogram of Figure 7 shows again that the counts of $\mathrm{Na}^{+}$ group are higher with $\mathrm{KH}$ waves.

\section{Discussion}

Figures 6 and 7 show some differences between the $\mathrm{KH}$ and non-KH events that raise the following questions: Why are differences in the properties of $\mathrm{Na}^{+} \mathrm{PSD}$ observed only in the magnetosphere between $\mathrm{KH}$ and non-KH cases? Why the counts are higher in $\mathrm{KH}$ and MSP regions during $\mathrm{KH}$ events than non- $\mathrm{KH}$ events?

First of all, we note that all data considered in this study were obtained in the nightside-duskside region. In that region, we expect two different populations of ions. The first one is composed of ions incoming from the magnetotail, that likely experience energization up to several $\mathrm{keV}$ due to nonadiabatic motion in the magnetotail (Delcourt et al., 2003). Because of their large Larmor radii, most of these energetic ions are lost at the magnetopause before gaining access to the dayside magnetosphere. The other one is composed of ions originating directly from the exosphere which does not circulate in the magnetotail. These ions at low energies (approximately a few eV) cannot be observed by FIPS that has a lower limit of $100 \mathrm{eV}$. In this study, we did not analyze the data in the dayside where the pickup ions are considered as the dominant population because the counts in the FIPS instrument were too low to provide the properties of planetary ions. On the other hand, in the region of our interest, the nightside-duskside magnetospheric flanks, FIPS detected enough counts to provide the information. Thus, ions coming from the magnetotail can be considered as the dominant population. Under that assumption, the large differences in the properties of the $\mathrm{Na}^{+}$PSD observed only in the magnetosphere can be understood if those high-energy ions are somehow decelerated when $\mathrm{KH}$ waves are recorded. According to the configuration that the $\mathrm{KH}$ instability likely occurs at planets' magnetopause, the $Z$ component of the magnetic field is dominant, and the plasma flows in both the magnetosheath and magnetosphere. In general, the particle acceleration occurs when the field changes rapidly 


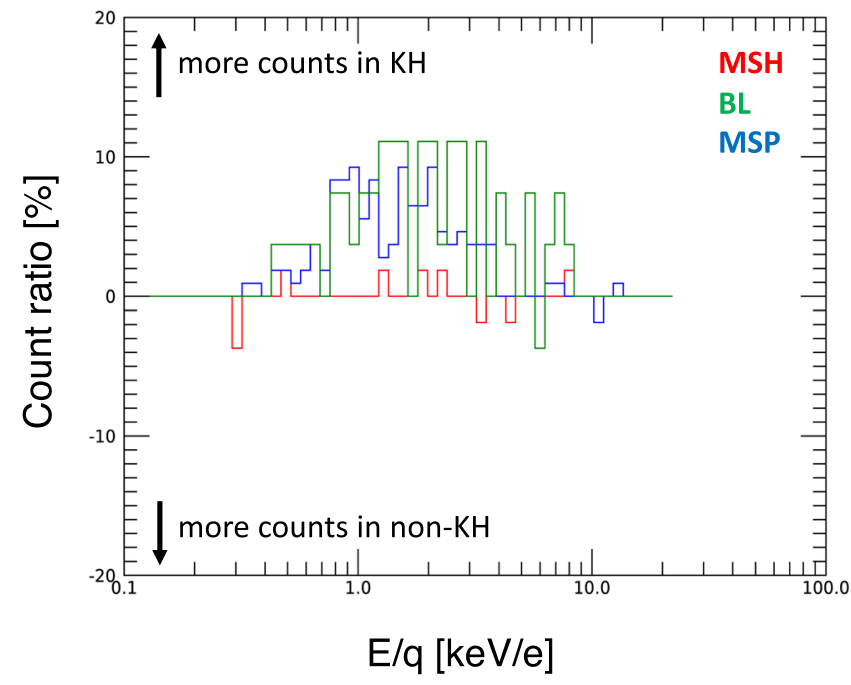

Figure 7. Histogram of the $\mathrm{Na}^{+}$group count ratio between $\mathrm{KH}$ and non-KH events for the 16 paired $\mathrm{KH}$ events identified in the text. The format of the figure is the same as the one of Figure 4. in the respect to the ion gyration motion, such as the magnetic reconnection and the relatively small curvature of the magnetic field line. However, in this statistical analysis, we see that the energy distribution of $\mathrm{Na}^{+}$group changed in the sense of deceleration when $\mathrm{KH}$ waves are recorded. The most possible mechanism to understand this deceleration is due to the electric field variation occurring during the development of the KH instability. Aizawa et al. (2018) have discussed the effect of the electric field during the growth of the $\mathrm{KH}$ instability on the particle acceleration. Due to the $Z$ component of the magnetic field and plasma flows, the electric field calculated by Ohm's law lies in the equatorial plane, and it can nonadiabatically accelerate or decelerate ambient planetary ions depending on both the initial particle energy and the direction between the ion motion and electric field vectors. They show that, when the ion has similar energy as the energy calculated by fields in the magnetosheath, they are possibly decelerated when ions are moving in the opposite direction of the electric field. The solar wind speed for each $\mathrm{KH}$ event can be estimated from the FIPS data to be between 360 and $490 \mathrm{~km} / \mathrm{s}$, equivalently between 1.5 and $3.0 \mathrm{keV}$. These values are consistent with the energy range where the different properties of the $\mathrm{Na}^{+}$PSD are observed between $\mathrm{KH}$ and non-KH events. For example, if an ion has $3.0 \mathrm{keV}$ in the magnetosphere and moving toward the dayside magnetosphere, its gyration motion has opposite rotation than that of $\mathrm{KH}$ vortices. This ion can be decelerated and lose its energy, and it will be the same level as the energy in magnetosheath or less, meaning they could be observed in the energy below $3.0 \mathrm{keV}$. Therefore, although we could not obtain detailed electric field components from MESSENGER observations, we suggest that the deceleration of ions due to their interactions with the $\mathrm{KH}$ vortices can explain the difference observed in the properties of the $\mathrm{Na}^{+}$PSD.

The higher $\mathrm{Na}^{+}$counts during $\mathrm{KH}$ cases have also been observed in the statistical study. This feature can be explained in two ways. The first is that observed ions were initially picked up ions that were subsequently accelerated by the development of the KH instability. Because of the limited energy range of FIPS, ions with low energy, like just created and escaped from the exosphere $(<10 \mathrm{eV})$, cannot be detected. However, if such ions are energized due to the development of the $\mathrm{KH}$ instability, they may have enough energy to be detected

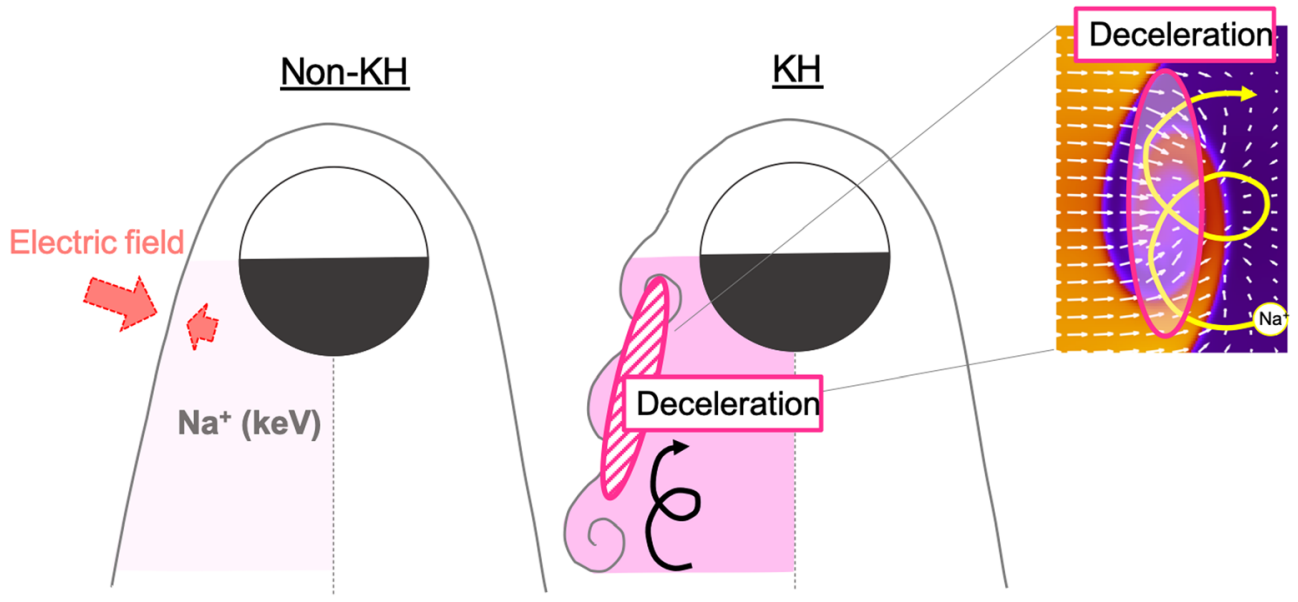

Figure 8. Illustration of the particle behavior in the vicinity of $\mathrm{KH}$ vortices. The different tint of pink in the region of interest shows the fact that the higher counts were observed in the $\mathrm{KH}$ case. Planetary ions observed in the magnetosphere seem not to escape to the magnetosheath due to the convection electric field shown in the right panel, which is the same for both the $\mathrm{KH}$ and non-KH cases. However, the electric field created during the development of the $\mathrm{KH}$ instability causes deceleration of ions. Although the zoomed figure on the right is the electric field (white arrows) under a pure northward IMF case, the ion experiences the larger electric field pushing back the ion against its motion, appearing as a deceleration in the $\mathrm{Na}^{+}$PSD signature. 
by FIPS and thus can also account for the observed increase in $\mathrm{Na}^{+}$counts. In this case, the properties of the $\mathrm{Na}^{+}$PSD are expected to be changed in the sense of acceleration in the energy range around a few keV. However, the figure shows the deceleration signature; thus, it is stressed here that the picked up ions are not a dominant population. The second explanation is that the presence of a significant population of $\mathrm{Na}^{+}$ ions at all energies in the magnetosphere may itself enhance the development of the KH instability, as suggested by Fujimoto et al. (2007). In any case, those ions seem not to escape from the magnetosphere and stay inside the magnetosphere. Under the ideal northward IMF, the large convection electric field in the magnetosheath is pointing toward the magnetopause and that in the magnetosphere also points toward the magnetopause. This configuration prevents ions from escaping from the magnetosphere.

\section{Summary}

Analysis of MESSENGER FIPS data in the magnetospheric flanks under northward IMF reveals two significant differences for the properties of the $\mathrm{Na}^{+}$PSD data between $\mathrm{KH}$ and non-KH events: (1) a deceleration signature for ions with energy above $2.0 \mathrm{keV} / \mathrm{e}$ in the presence of $\mathrm{KH}$ waves and (2) higher counts in FIPS data when the KH waves are observed. The dominant ion population that FIPS observed in the region of this study seems to be ions originating from the magnetotail with the energy a few keV. They are decelerated by the $\mathrm{KH}$ structures due to the opposite gyromotion of ions in $\mathrm{KH}$ waves electric field, and they stagnate inside the magnetosphere because of the convection electric field (see Figure 8). On the other hand, the very small acceleration signatures around $1 \mathrm{keV} / \mathrm{e}$ are observed. Although this is almost negligible, it may be related to the acceleration of picked up ions from the exosphere that is not the dominant population in the region of interest. By comparing the counts of planetary ions in FIPS, we see clear increases for $\mathrm{KH}$ events between $\mathrm{KH}$ and non- $\mathrm{KH}$ events. We suggest that, following their deceleration, these ions stagnate in this region of space after convection from the distant tail due to the electric field.

The understanding of those ions in the magnetospheric flanks is important for a better understanding of plasma convection in the magnetosphere. However, due to the limited instruments of MESSENGER to observe the plasma environment at Mercury, it is difficult to get the electric field data. In order to examine the hypothesis we suggested, further investigation will be required. The BepiColombo mission, the joint project of the European Space Agency and the Japan Aerospace Exploration Agency, has the full package of instruments sophisticated to observe Mercury's plasma environment.

\section{Data Availability Statement}

The FIPS and MAG data are available on the Planetary Data System (https://pds.nasa.gov).

\section{Acknowledgments}

The work of S. Aizawa was supported by the International Joint Graduate Program in Earth and Environmental Sciences (GP-EES). French coauthors acknowledge the support of CNES for the BepiColombo mission.

\section{References}

Aizawa, S., Delcourt, D., \& Terada, N. (2018). Sodium ion dynamics in the magnetospheric flanks of Mercury. Geophysical Research Letters, 45, 595-601. https://doi.org/10.1002/2017GL076586

Chen, S.-H., \& Kivelson, M. G. (1993). On nonsinusoidal waves at the Earth's magnetopause. Geophysical Research Letters, 20(23), 2699-2702. https://doi.org/10.1029/93GL02622

Delcourt, D. C., Grimald, S., Leblanc, F., Berthelier, J.-J., Millilo, A., Mura, A., et al. (2003). A quantitative model of the planetary Na ${ }^{+}$ contribution to Mercury's magnetosphere. Annales de Geophysique, 21(8), 1723-1736. https://doi.org/10.5194/angeo-21-1723-2003

Elphic, R. C., \& Ershkovich, A. I. (1984). On the stability of the ionopause of Venus. Journal of Geophysical Research, 89(A2), 997-1002. https://doi.org/10.1029/JA089iA02p00997

Fujimoto, M., Baumjohann, W., Kabin, K., Nakamura, R., Slavin, J. A., Terada, N., \& Zelenyi, L. (2007). Hermean magnetosphere-solar wind interaction. Space Science Reviews, 132(2-4), 529-550. https://doi.org/10.1007/s11214-007-9245-8

Fujimoto, M., \& Terasawa, T. (1994). Anomalous ion mixing within an MHD scale Kelvin-Helmholtz vortex. Journal of Geophysical Research, 99(A5), 8601-8613. https://doi.org/10.1029/93JA02722

Fujimoto, M., Tonooka, T., \& Mukai, T. (2003). In P. T. Newell \& T. Onsager (Eds.), Vortex-like fluctuations in the magnetotail flanks and their possible roles in plasma transport, the Earth's low-latitude boundary layer, Geophysical Monograph Series (Vol. 133, pp. 241-251). Washington, D. C: American Geophysical Union. https://doi.org/10.1029/133GM24

Gershman, D. J., Raines, J. M., Slavin, J. A., Zurbuchen, T. H., Sundberg, T., Boardsen, S. A., et al. (2015). MESSENGER observations of multiscale Kelvin-Helmholtz vortices at Mercury. Journal of Geophysical Research: Space Physics, 120, 4354-4368. https://doi.org/ 10.1002/2014JA020903

Gershman, D. J., Slavin, J. A., Raines, J. M., Zurbuchen, T. H., Anderson, B. J., Korth, H., et al. (2013). Magnetic flux pileup and plasma depletion in Mercury's subsolar magnetosheath. Journal of Geophysical Research: Space Physics, 118, 7181-7199. https://doi.org/10.1002/ 2013JA019244

Gingell, P. W., Sundberg, T., \& Burgess, D. (2015). The impact of a hot sodium ion population on the growth of the Kelvin-Helmholtz instability in Mercury's magnetotail. Journal of Geophysical Research: Space Physics, 120, 5432-5442. https://doi.org/10.1002/ 2015JA021433 
Hasegawa, H., Fujimoto, M., Phan, T.-D., Reme, H., Balogh, A., Dunlop, M. W., et al. (2004). Transport of solar wind into Earth's magnetosphere through rolled-up Kelvin-Helmholtz vortices. Nature, 430(7001), 755-758. https://doi.org/10.1038/nature02799

Hwang, K.-J., Kuznetsova, M. M., Sahraoui, F., Goldstein, M. L., Lee, E., \& Parks, G. K. (2011). Kelvin-Helmholtz waves under southward interplanetary magnetic field. Journal of Geophysical Research, 116, A08210. https://doi.org/10.1029/2011JA016596

Liljeblad, E., Karlsson, T., Sundberg, T., \& Kullen, A. (2016). Observations of magnetospheric ULF waves in connection with the Kelvin-Helmholtz instability at Mercury. Journal of Geophysical Research: Space Physics, 121, 8576-8588. https://doi.org/10.1002/ 2016JA023015

Liljeblad, E., Sundberg, T., Karlsson, T., \& Kullen, A. (2014). Statistical investigation of Kelvin-Helmholtz waves at the magnetopause of Mercury. Journal of Geophysical Research: Space Physics, 119, 9670-9683. https://doi.org/10.1002/2014JA020614

Miura, A. (1984). Anomalous transport by magnetohydrodynamic Kelvin-Helmholtz instabilities in the solar wind-magnetospheric interaction. Journal of Geophysical Research, 89(A2), 801-818. https://doi.org/10.1029/JA089iA02p00801

Mozer, F. S., Hayakawa, H., Kokubun, S., Nakamura, M., Okada, T., Yamamoto, T., \& Tsuruda, K. (1994). The morningside low-latitude boundary layer as determined from electric and magnetic field measurements on Geotail. Geophysical Research Letters, 21(25), 2983-2986. https://doi.org/10.1029/94GL01296

Nakamura, T. K. M., \& Fujimoto, M. (2006). Magnetic reconnection within MHD-scale Kelvin-Helmholtz vortices triggered by electron inertial effects. Advances in Space Research, 37(3), 522-526. https://doi.org/10.1016/j.asr.2005.01.057

Nykyri, K., \& Otto, A. (2001). Plasma transport at the magnetospheric boundary due to reconnection in Kelvin-Helmholtz vortices. Geophysical Research Letters, 28(18), 3565-3568. https://doi.org/10.1029/2001GL013239

Paral, J., \& Rankin, R. (2013). Dawn-dusk asymmetry in the Kelvin-Helmholtz instability at Mercury. Nature Communications, 4(1), 1645. https://doi.org/10.1038/Ncomms2676

Raines, J. M., Gershman, D. J., Zurbuchen, T. H., Sarantos, M., Slavin, J. A., Gilbert, J. A., et al. (2013). Distribution and compositional variations of plasma ions in Mercury's space environment: The first three Mercury years of MESSENGER observations. Journal of Geophysical Research: Space Physics, 118, 1604-1619. https://doi.org/10.1029/2012JA018073

Shue, J.-H., Chao, J. K., Fu, H. C., Russell, C. T., Song, P., Khurana, K. K., \& Singer, H. J. (1997). A new functional form to study the solar wind control of the magnetopause size and shape. Journal of Geophysical Research, 102(A5), 9497-9511. https://doi.org/10.1029/ 97JA00196

Slavin, J. A., Acuña, M. H., Anderson, B. J., Baker, D. N., Benna, M., Gloeckler, G., et al. (2008). Mercury's magnetosphere after MESSENGER's first flyby. Science, 321(5885), 85-89. https://doi.org/10.1126/science.1159040

Slavin, J. A., Anderson, B. J., Zurbuchen, T. H., Baker, D. N., Krimigis, S. M., Acuña, M. H., et al. (2009). MESSENGER observations of Mercury's magnetosphere during northward IMF. Geophysical Research Letters, 36, L02101. https://doi.org/10.1029/2008GL036158

Slavin, J. A., Lepping, R. P., Wu, C.-C., Anderson, B. J., Baker, D. N., Benna, M., et al. (2010). MESSENGER observations of large flux transfer events at Mercury. Geophysical Research Letters, 37, L02105. https://doi.org/10.1029/2009GL041485

Sonnerup, B. U., \& Cahill, L. J. (1967). Magnetopause structure and attitude from Explorer 12 observations. Journal of Geophysical Research, 72(1), 171-183. https://doi.org/10.1029/JZ072i001p00171

Sundberg, T., Boardsen, S. A., Slavin, J. A., Anderson, B. J., Korth, H., Zurbuchen, T. H., et al. (2012). MESSENGER orbital observations of large-amplitude Kelvin-Helmholtz waves at Mercury's magnetopause. Journal of Geophysical Research, 117, A04216. https://doi.org/ $10.1029 / 2011 \mathrm{JA} 017268$

Sundberg, T., Boardsen, S. A., Slavin, J. A., Blomberg, L. G., Cumnock, J. A., Solomon, S. C., et al. (2011). Reconstruction of propagating Kelvin-Helmholtz vortices at Mercury's magnetopause. Planetary and Space Science, 59(15), 2051-2057. https://doi.org/10.1016/j.pss. 2011.05.008

Winslow, R. M., Anderson, B. J., Johnson, C. L., Slavin, J. A., Korth, H., Purucker, M. E., et al. (2013). Mercury's magnetopause and bow shock from MESSENGER Magnetometer observations. Journal of Geophysical Research: Space Physics, 118, 2213-2227. https://doi.org/ 10.1002/jgra.50237

Yan, G. Q., Mozer, F. S., Shen, C., Chen, T., Parks, G. K., Cai, C. L., \& McFadden, J. P. (2014). Kelvin-Helmholtz vortices observed by THEMIS at the duskside of the magnetopause under southward interplanetary magnetic field. Geophysical Research Letters, 41, 4427-4434. https://doi.org/10.1002/2014GL060589 